\title{
Design and Analysis of a Dual-Stator Spoke-Type Linear Vernier Machine for Wave Energy Extraction
}

\author{
Salman Khaliq* and Byung-il Kwon ${ }^{\dagger}$
}

\begin{abstract}
In this paper, a dual-stator, spoke-type linear vernier machine (DSSLVM) for wave energy extraction application was proposed. This machine is capable of producing a competitively high thrust force and force density at a low operation speed in direct drive systems. The operation principal and working of the proposed DSSLVM were studied. The stator core height is adjusted to improve the overall force density of the proposed machine while reducing the force ripple. To evaluate the advantages of the proposed DSSLVM, the main performance was compared with that of a recently developed linear primary permanent magnet vernier machine (LPPMVM). The proposed machine exhibited greater thrust force and force density, an improved power factor and lower force ripple with the same permanent magnet (PM) volume compared to the LPPMVM.
\end{abstract}

Keywords: Double stator, Thrust force density, Linear machine, Spoke-type magnet, Low speed, Direct drive

\section{Introduction}

In recent years, research has emphasized the extraction of energy from renewable energy resources such as wind turbines, hydropower, solar and ocean energy as they are comparatively cheaper and environmentally friendly processes. Ocean energy in the form of waves is a significant source of renewable energy. There are mainly two methods for capturing and converting the wave energy of the sea. The first one utilizes rotating electrical machines as with the generation of other forms of renewable energy. It uses a complex intermediate gear to convert the slow moving wave's linear motion to high-speed rotating motion to be utilized in rotating machines. Thus, the overall system suffers from high maintenance and lower reliability. The other method is to directly drive the machine from the reciprocating motion, i.e., linear generators. These machines act as a direct drive system, eliminating mechanical gears and reducing the energy conversion steps from rotary to linear motion, which in turn reduces the maintenance costs and increases the efficiency [1]. Consequently, the overall cost of the system is reduced with improved reliability.

However, permanent magnet (PM) linear machines suffer from a bulky size due to a large number of winding poles to fulfill the low-speed operation requirement, which decreases the power density of the machine. Recently, magnetic gears (MGs) have been used to resolve this issue due to their flux-modulating capability, which converts the slow moving flux of PMs to higher speeds. Besides that, these MGs have better reliability due to less physical

$\dagger$ Corresponding Author: Dept. of Electronic Systems Engineering, Hanyang University, Korea. (bikwon@hanyang.ac.kr)

* Dept. of Electronic Systems Engineering, Hanyang University, Korea. (salman.khaliq@hotmail.com)

Received: December 14, 2015; Accepted: June 1, 2016 oscillation, acoustic noise and maintenance costs compared to mechanical gears. Based on this principle, some highperformance magnetic gears with high power density have been developed $[2,3]$.

Furthermore, a pseudo direct drive machine was proposed, which utilizes MGs to convert the low-speed magnetic flux to high speed for electrical generation [4]. However, this machine suffers from manufacturing aspect due to having three air gaps and two moving parts. To resolve this issue, a generic torque-maximizing machine having only one air gap and a higher torque density is developed, and a linear version of it has been developed since $[5,6]$. However, these machines have magnets on the translator which makes their mechanical and thermal design more unstable and less robust [7]. It also increases the manufacturing cost of such machines. Linear vernier hybrid machines (LVHs) have been developed to resolve this issue. These machines have magnets on the stator and work on the principle of flux reversal [8]. These machines were further researched to improve the force density in single-stator linear primary permanent magnet vernier machines (LPPMVMs) and dual-stator higher temperature superconducting (HTS-LPPMVM) $[9,10]$.

However, linear vernier machines mentioned above suffer from a low power factor due to longitudinal end effects of linear structure and vernier nature. Which requires a high rated converter for the machine operation and increases the overall cost of the system $[11,12]$. To resolve this problem, a flux focusing spoke-type design was proposed in [13]. It effectively increases the flux density value in the air gap, which improves the power factor of the machine along with the power density through a dual-stator compact design. However, the linear version of this model has not yet been proposed. 
In this paper, a new topology of the linear stator vernier machine, named the dual-stator spoke-type linear vernier machine (DSSLVM), was proposed for high force density with an improved power factor. This machine uses PMs in the translator. These magnets are magnetized in the axial or horizontal direction to operate with both stators simultaneously. The spoke-type PM arrangement in the translator increases the effective utilization of the magnet's flux and generates a high flux density in the air gap. The proposed machine is designed as a flat type with similar stator dimensions and the same air gap length, which is different in the tubular design i.e. inner and the outer stator diameter. Based on the number of winding pole pairs and the number of teeth on stator and translator pole pairs, this machine is able to generate a higher force density at a low speed. In addition, the rectangular magnets were easily magnetized in the horizontal direction, which reduces the overall cost of the machine.

To clearly identify the advantages of the proposed DSSLVM, its performance was compared with that of a recently developed LPPMVM of [14] while keeping the overall PM volume and slot current density constant. In Section II, configurations of the proposed DSSLVM and LPPMVM are discussed. Section III illustrates the working and design principle of the proposed machine. In Section IV, the electromagnetic performance of the proposed DSSLVM is compared to that of LPPMVM via timestepping finite element analysis (FEM). Finally, the conclusion is presented in Section V.

\section{Configuration of the Proposed DSSLVM}

Fig. 1 shows the configuration of the recently developed LPPMVM. Its laminated iron stator core consists of six primary teeth and each tooth surface was mounted by five PMs, magnetized in the perpendicular and adjacent alternate directions as a surface-type PMVM. Distributed winding was adopted, and coils were wound across the primary teeth. Its translator is composed of iron with a slotted structure.

The proposed DSSLVM consists of two stators and one translator with spoke-type magnets inserted as shown in Fig. 2. Stator slots were designed as a split pole type instead of an open slot type in order to increase the housing space for the winding [15]. Each stator was divided into six pole shoes, each of which was further divided into three

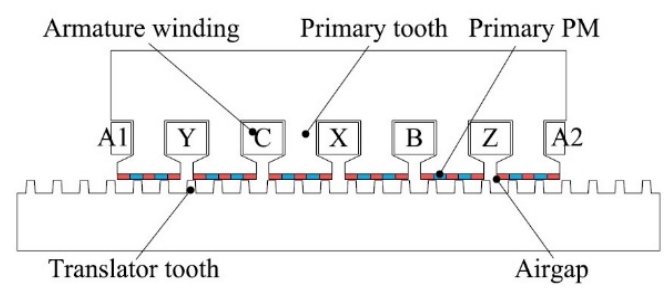

Fig. 1. Configuration of the basic LPPMVM

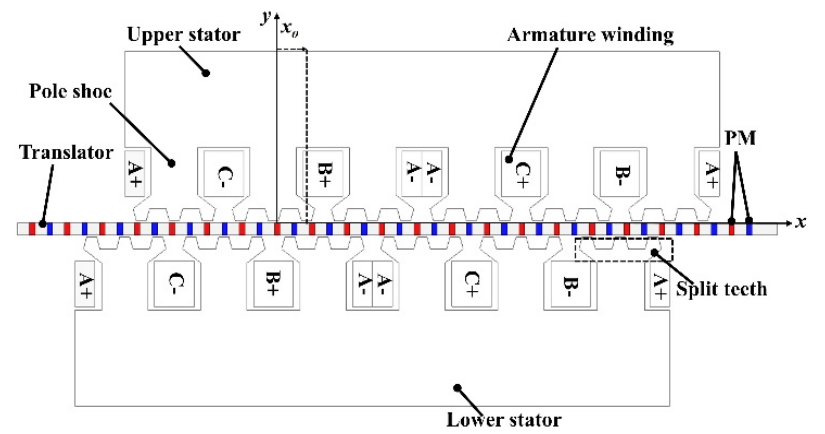

Fig. 2. Configuration of the proposed DSSLVM

split teeth that were used to modulate the flux of the translator PMs in the machine. Distributed coils were wound across the pole shoes. Windings of both stators can be connected in series utilizing only one inverter, as in a normal PM machine, or can be controlled separately according to the requirement. The two stators have the same construction, and their positions are maintained unaligned by one-half of a slot pitch in order to more effectively utilize the translator PM flux [13]. The ratio of the split tooth tip to the split tooth pitch $\tau_{\mathrm{s}}$ was maintained at 0.3 in order to produce the maximum thrust force, whereas the split tooth root to tooth pitch ratio was maintained at 0.5 as it does not have a significant effect on the output of the machine [16]. The proposed DSSLVM contained one translator composed of a flat iron core with a spoke-type array of inserted PMs. The heights of the translator core and PMs were kept the same. The PMs were magnetized in the horizontal direction, and the flux operated simultaneously with both stators. In contrast, this flux only goes through half of the machine in conventional dual-stator surface-mounted PM machines. Spoke-type magnets in the translator focus the flux more effectively in the air gap and decrease the leakage flux, resulting in a comparatively higher air gap flux density [13].

\section{Working and Design Principle}

The LPPMVM operates on the principle of vernier hybrid machines (VHMs), based on the flux reversal phenomenon in normal PM machines [9]. The flux linkage to the stator coils is maximized when the translator teeth are fully aligned with the magnet poles and is minimal at the unaligned position. The proposed DSSLVM operates on the principle of the "magnetic gearing effect," in which the flux of the magnets in the machine is modulated by a slotted structure in order to interact with a smaller number of pole pair magneto motive force (MMF). This principle can be illustrated by the equation given below:

$$
P_{p m}=N_{s} \pm p
$$

where $P_{p m}$ is the translator pole pair number, $N_{s}$ is the 
number of stator split teeth, and $p$ is the winding pole pair at the stator. The air gap magnetic flux density is directly proportional to the PM pole pairs in the vernier machine. This air gap flux density is then modulated by the variable permeance of the split tooth structure of the pole shoes to produce a high-speed flux with $p$ pole pairs, which is the same as the designed winding pole pair. This phenomenon enables the flux of a greater number of PM poles of the translator to interact with the flux produced by the smaller number of stator-wound pole pairs, as in the presented case. Spoke-type translator PMs magnetized in the horizontal direction focus the flux more efficiently in the air gap utilizing its maximum potential. Therefore, the cost of utilizing highly sintered $\mathrm{NdFeB}$ magnets can be reduced by decreasing the volume used in the machine [13].

Magnetic field analysis of the proposed DSS-LVM was performed with the so called unit block approach [17]. The permeance function is described as the permeance per unit area, $P(x)$, and is calculated as

$$
P(x)=P_{0}+(-1)^{j} \sum_{m=1}^{\infty} P_{m} \cos \left(m N_{s} \frac{2 \pi}{L} x\right)
$$

where $j$ is the number of slot shifts for short pitch winding, $P o$ is the average permeance of the air gap and $P m$ is the amplitude of mth harmonic. $L, x$ and $N s$ are the translator active length, distance moved in the horizontal direction and total number of smaller slots on the pole shoes of one stator.

The new proposed machine demonstrates permeance variation due to slots in the pole shoes and the spoke-type structure of the translator. Therefore, to calculate the permeance function, the carter factor is added to consider the effects of all the variations mentioned above [18], and [19]. Therefore, assuming infinite permeability for the iron core and using Ampere's law, $F_{\text {gap }}$, which is the average of the fundamental component of the MMF of both air gaps, can be calculated as

$$
F_{\text {gap }}=\frac{B_{r} g_{m}}{\mu_{m}} /\left(2+\frac{P_{0} g_{m}}{4 h_{m} \mu_{0}} \tau_{r}\right)
$$

where $B_{r}, g_{m}$ and $h_{m}$ are the magnet residual flux density, magnet thickness in the horizontal direction and the magnet height, respectively. $\mu_{o}$ is the relative permeability of the air, $\mu_{m}$ is the magnet permeability and $\tau_{r}$ is the magnet pole pitch of the translator. Furthermore, the air gap flux density, $B_{P M}(x)$, is calculated considering only the lower-order component of MMF, $n=1$ and the permeance coefficients of $m=0,1$ which can be described as

$$
\begin{aligned}
B_{P M}(x) & =F_{\text {gap }}(x) P(x) \\
& =\frac{4}{\pi} F_{\text {gap }} P_{0} \cos \left(P_{P M} \frac{2 \pi}{L}\left(x-x_{m}\right)\right)+(-1)^{j} \frac{2}{\pi} F_{g a p} P_{1}
\end{aligned}
$$

$$
\cos \left(\left(P_{P M}-N_{s}\right) \frac{2 \pi}{L} x-P_{P M} \frac{2 \pi}{L} x_{m}\right)+B_{h a r}
$$

The first term of (4) is due to the direct effect of PM and have a shorter wave length while the second term of the above expression has a long wavelength. It interacts with the flux produced by the stator winding currents, which has a $p$ pole pair, to produce a steady force as also described in (1). This is also known as the gearing effect in vernier machines. This analytically calculated air gap flux density distribution is verified with the transient 2D FEM, as shown in Fig. 3. The end effect is ignored in this analysis as those are notably higher only in high speed linear machines while the proposed model speed is low i.e. $1 \mathrm{~m} / \mathrm{s}$ [12].

Currently, there are two kinds of topologies for linear machines, short travel progressive or oscillatory, and long straight travel with a longer translator or stator as compared to the other. In the former case, the length difference between the translator and stator cores is not much as these machines are used for short travel applications, e.g., pen recorders, free power pistons or wave energy extraction [20, 21]. The latter case topology is more suitable for long linear motion, e.g. maglev. The proposed DSSLVM falls into the former category. In the DSSLVM, extra PM poles are inserted in the translator according to prevailing motion

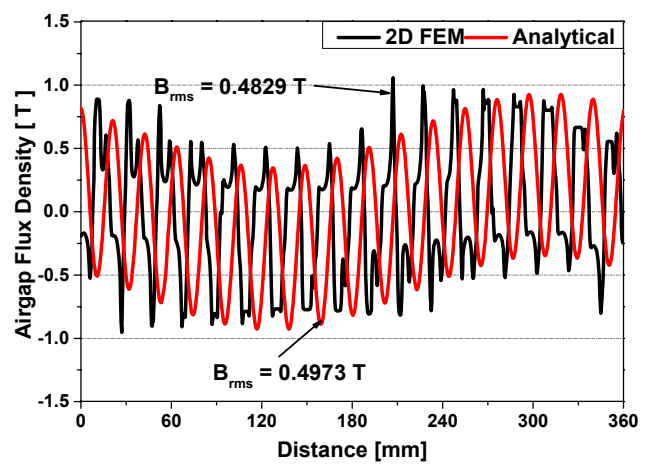

Fig. 3. Air gap flux density distribution according to $2 \mathrm{D}$ FEM and the analytical method

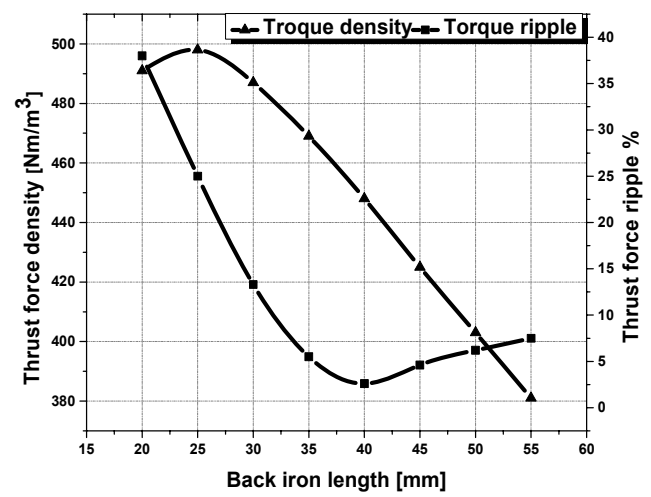

Fig. 4. Thrust force and thrust force ripple vs. stator core height 


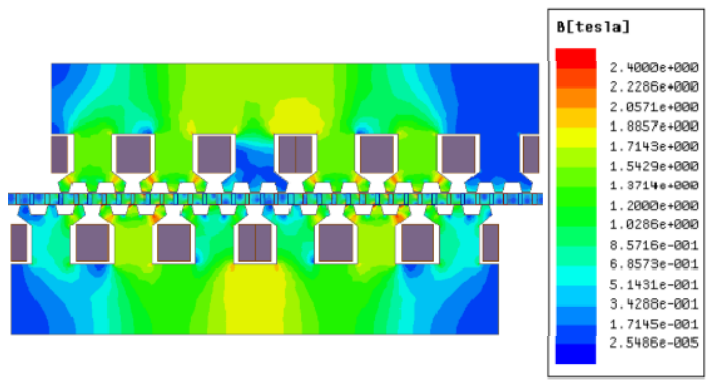

Fig. 5. Flux density in the DSSLVM with a load condition

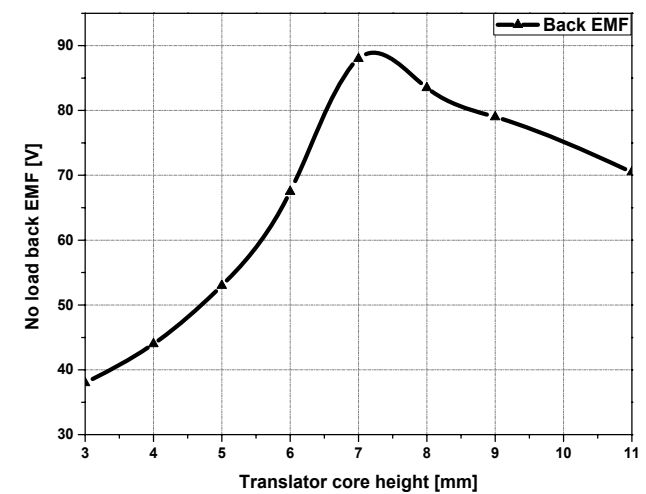

Fig. 6. Load back EMF versus translator core height

conditions on the seashore. The same magnet volume as for the LPPMVM is used, while the active volume of the magnets, i.e., the volume of working magnets at a given moment for the both stators, is $67 \%$ of the PMs utilized in LPPMVM.

Furthermore, the back iron height of the stator core was adjusted and its effect was analyzed on the force ripple and force density of the machine. The analysis is illustrated in Fig. 4. The split tooth height and width are also adjusted to avoid any saturation and to decrease the detent force in the machine. The flux density distribution at the rated load case is shown in Fig. 5, where the maximum flux density in the stator and translator cores of the proposed DSSLVM is less than $1.8 \mathrm{~T}$.

Since the flux density distribution presented in Fig. 5 shows a relatively low-flux density in the translator core in the proposed machine, the translator core height is analyzed to determine the effect on the back EMF. The height of the core is varied while maintaining the same magnet height and mechanical air gap length. Fig. 6 shows that the maximum no-load back EMF is achieved at approximately $7 \mathrm{~mm}$, which is similar to the magnet height.

\section{Electromagnetic Comparison}

The proposed DSSLVM is compared with the LPPMVM of [16] to determine the advantages of the proposed design. Design parameters of both machines are described in Table 1 .
Table 1 Design parameters of LPPMVM and DSSLVM

\begin{tabular}{|c|c|c|c|}
\hline Items & Unit & LPPMVM $^{[16]}$ & DSS LVM \\
\hline Rated current & $\mathrm{A}$ & \multicolumn{2}{|l|}{13} \\
\hline Magnet remanence & $\mathrm{T}$ & \multicolumn{2}{|c|}{1.2} \\
\hline Air gap length & $\mathrm{mm}$ & 1 & $2 * 1$ \\
\hline No. of turns per phase & - & 142 & 131 \\
\hline Magnetic gear ratio & - & \multicolumn{2}{|l|}{17} \\
\hline No. of teeth on one pole shoe & - & - & 3 \\
\hline Winding pole pairs on one stator & - & \multicolumn{2}{|l|}{1} \\
\hline No. of pole shoes on one stator & - & \multicolumn{2}{|l|}{6} \\
\hline Thickness of PM & $\mathrm{mm}$ & 4 & 3.2 \\
\hline Height of one stator & $\mathrm{mm}$ & 101 & 66.5 \\
\hline Height of translator core & $\mathrm{mm}$ & 56 & 7 \\
\hline Active length of one stator & $\mathrm{mm}$ & \multicolumn{2}{|c|}{360} \\
\hline Active length of the machine & $\mathrm{mm}$ & 360 & 383 \\
\hline Active PM volume & $\mathrm{cm}^{3}$ & 120 & 80 \\
\hline
\end{tabular}

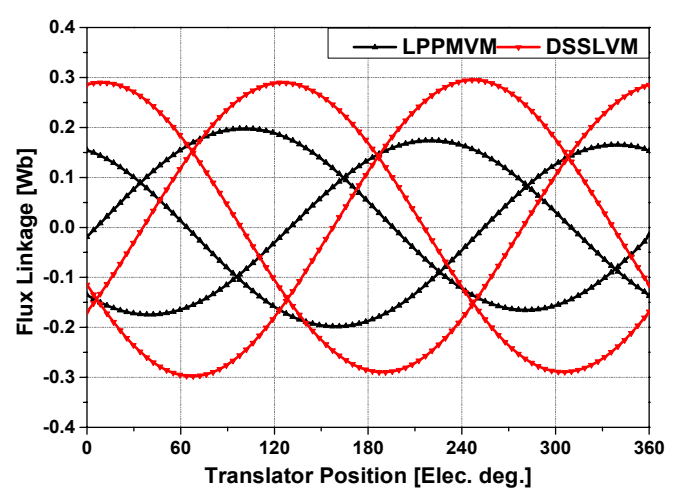

Fig. 7. Comparison of flux linkage

Both machines have the same slot current density, although the proposed DSSLVM utilizes a less active magnet volume compared to LPPMVM due to a shorter translator core height design. The number of turns per phase is modified for the proposed machine while maintaining the same slot current density. The slot area and the number of turns in the proposed machine are modified to distribute turns equally on both stators, whereas in LPPMVM they are on one stator. The 2D transient FEM is performed to compare the performance of both machines.

Fig. 7 compares the no-load flux linkage of both machines. The maximum flux linkage value in DSSLVM is $0.3 \mathrm{~Wb}$, which is $50 \%$ higher than the $0.2 \mathrm{~Wb}$ of LPPMVM due to the flux focusing design of the proposed machine, which more efficiently directs the PM flux to the stator coils.

The no-load back EMF values of both machines, which can be obtained by differentiating the no-load flux linkage, are compared in Fig. 8. The peak values of the back EMF in the LPPMVM and the proposed DSSLVM are $60 \mathrm{~V}$ and $86 \mathrm{~V}$, respectively. Specifically, the proposed DSSLVM produces a no-load back EMF $43 \%$ larger than that of the LPPMVM while utilizing a $33 \%$ less active magnet volume.

The output thrust force in the linear machine is expressed as 


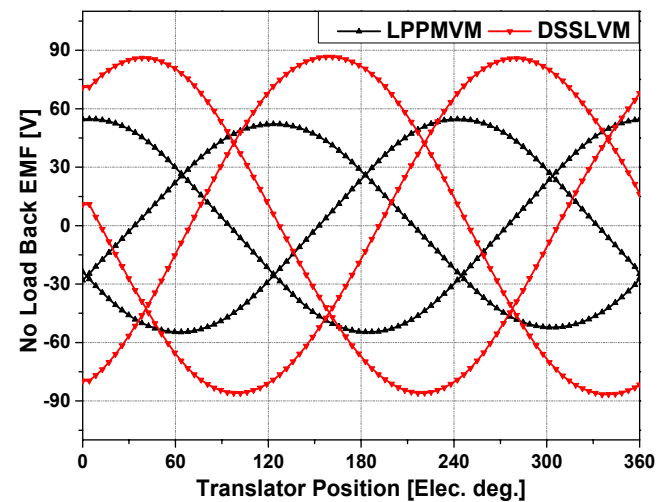

Fig. 8. Comparison of no-load back EMF

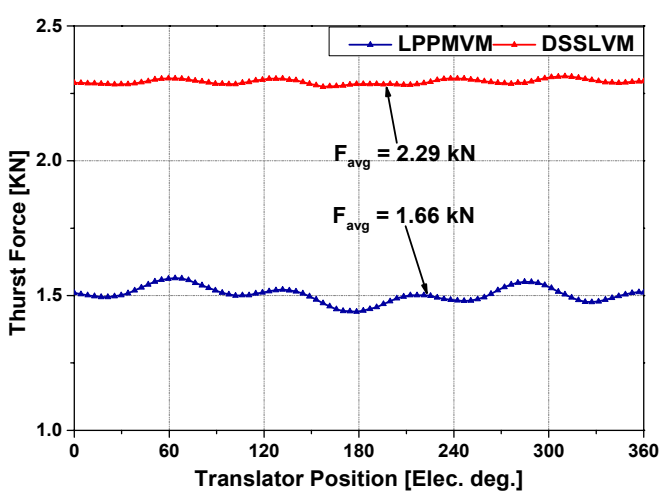

Fig. 9. Comparison of thrust force

$$
F_{e m}=\left(e_{a} i_{a}+e_{b} i_{b}+e_{c} i_{c}\right) / v
$$

Where $e_{a}, e_{b}$ and $e_{c}$ are the rms phase back emf of three phase and $i_{a}, i_{b}$ and $i_{c}$ are the rms values of armature current. Fig. 9 compares the average value of the thrust force between the proposed DSSLVM and LPPMVM, which demonstrated respective values of 2293 $\mathrm{N}$ and $1662 \mathrm{~N}$. This demonstrates that the average thrust force of the proposed DSSLVM is 38\% higher than that of LPPMVM. The force ripple in the proposed machine is also lower, i.e., $1.6 \%$ as compared to 3\% in LPPMVM [16]. The thrust force density of the proposed machine, which is the ratio of the average thrust force to active volume of the machine, is also $57 \%$ greater than that of LPPMVM. This is mainly because of the dual-stator topology of the proposed DSSLVM with a higher overall output thrust force and adjusted back iron length. The larger flux density design of the DSSLVM also results in a higher detent force, as listed in Table 2. The detent force is $1.81 \%$ of the average thrust force in LPPMVM and 3.7\% in DSSLVM, which is mainly due to the spoke-type structure in the proposed machine.

Furthermore, spoke-type PM machines such as the proposed DSSLVM have different reluctance values in the $\mathrm{d}$ and q-axis directions, producing additional reluctance force compared to that of the surface-type PM machine. In general, the electromagnetic thrust force in spoke type PM machines is expressed as
Table 2 Transient FEA performance comparison

\begin{tabular}{c|c|c|c}
\hline Items & Unit & LPPMVM $^{[16]}$ & DSSLVM \\
\hline No-load back EMF & $\mathrm{V}$ & 60 & 86 \\
\hline Detent force & $\mathrm{N}$ & $30(1.8 \%)$ & $84(3.7 \%)$ \\
\hline Average thrust force & $\mathrm{kN}$ & 1.66 & 2.29 \\
\hline Thrust force ripple & $\mathrm{N}$ & $49(3 \%)$ & $38(1.6 \%)$ \\
\hline Thrust force density & $\mathrm{kN} / \mathrm{m}^{3}$ & 285 & 448 \\
\hline Power factor & - & 0.26 & 0.40
\end{tabular}

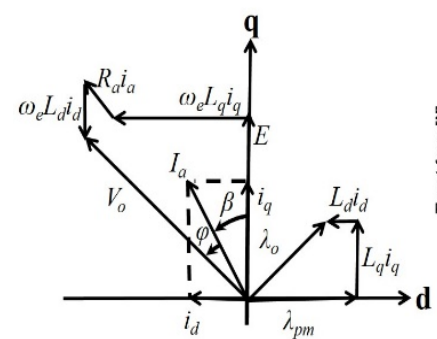

(a)

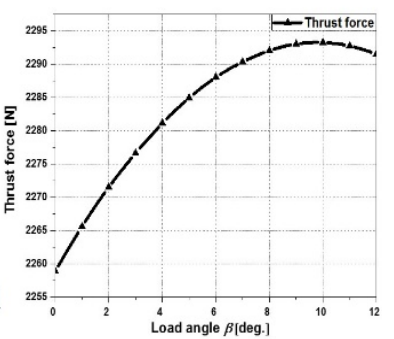

(b)
Fig. 10. Characteristic of IPM model: (a) Steady state vector diagram; (b) Thrust force vs load angle

$$
F_{e m}=\frac{3 p}{2}\left[\lambda_{p m} I_{a} \cos \beta+\frac{1}{2}\left(L_{d}-L_{q}\right) I_{a}^{2} \sin 2 \beta\right]
$$

Where $I_{a}$ is the peak value of the phase current, $\beta$ is the phase current angle measured from q- axis. $L_{d}$ and $L_{q}$ are the direct and $\mathrm{q}$ axis inductances. The first term in (6) is the force due to PM flux and the $2^{\text {nd }}$ term is due to reluctance variation in the machine. Therefore, to achieve the maximum thrust output, the excitation current is applied with a load angle $\beta$ related to the back EMF vector. Fig. 10(a) shows the steady state vector diagram in IPM machines while the variation in the thrust force output with respect to load angle $\beta$ is shown in Fig. 10(b). A maximum thrust force is obtained at a load angle of $9^{\circ}$, as illustrated in Fig. 10.

The proposed machine analysis suggests that DSSLVM is also capable of a high power factor due to its flux focusing design. The power factor of DSSLVM is compared with LPPMVM, while keeping all of the other parameters at similar values. The proposed machine exhibits a power factor of 0.4 compared to 0.26 for LPPMVM. This verifies the flux focusing design and better utilization of magnetic material in the proposed DSSLVM. However the overall lower value of the power factor in both machines is due to the larger air gap to pole pitch ratio and longitudinal end effects due to linear structure and vernier nature of the machines [11, 12]. Table 2 summarizes the transient FEA performance comparison of the proposed DSSLVM with LPPMVM.

\section{Conclusion}

In this paper, a new dual-stator topology for a linear 
machine was presented, which exhibits a considerably high thrust force and thrust force density for low-speed applications using spoke-type PMs. The back iron length of the stator core is adjusted to increase the force density and decrease the force ripple. The translator core height is also adjusted to maximize the back EMF. The advantages of the proposed DSSLVM were determined by comparing the FEM analysis results with those of a recently developed LPPMVM. The comparison suggests that the proposed machine has a greater no-load back EMF, force density, improved power factor and lower torque ripple than the presented LPPMVM, all while utilizing a lower active magnet volume. Only the detent force in the proposed machine is a little higher compared to LPPMVM due to the spoke-type structure of the proposed machine. Furthermore, the proposed DSSLVM has magnets on the translator, which makes the design less stable and more complex due to the manufacturing process compared to LPPMVM. However, utilizing the same PM volume as LPPMVM with a higher force density and improved quality of output power and power factor makes DSSLVM a more viable option for low-speed, high-thrust force applications, e.g. wave energy extraction.

\section{Acknowledgment}

This work was supported in part by the Human Resources Program in Energy Technology of the Korea Institute of Energy Technology Evaluation and Planning (KETEP), granted financial resource from the Ministry of Trade, Industry \& Energy, Republic of Korea. (No. 20154030200730), and in part by the National Research Foundation of Korea (NRF) grant funded by the Korea government (Ministry of Science) (No.NRF2013R1A2A2A01068022).

\section{References}

[1] Mueller, M.; Baker, N. J., "A low speed reciprocating permanent magnet generator for direct drive wave energy converters," in Power Electronics, Machines and Drives, 2002. International Conference on (Conf. Publ. No. 487), vol., no., pp. 468-473, 4-7 June 2002 doi:10.1049/cp:20020162.

[2] Atallah, K.; Howe, D., "A novel high-performance magnetic gear," in Magnetics, IEEE Transactions on, vol. 37, no. 4, pp. 2844-2846, Jul 2001 doi:10.1109/ 20.951324.

[3] Rasmussen, P. O.; Andersen, T. O.; Jorgensen, F. T.; Nielsen, O., "Development of a high-performance magnetic gear," in Industry Applications, IEEE Transactions on, vol. 41, no. 3, pp. 764-770, MayJune 2005 doi:10.1109/TIA.2005.847319.

[4] Wenlong Li; Chau, K. T.; Jiang, J.Z., “Application of Linear Magnetic Gears for Pseudo-Direct-Drive Oceanic Wave Energy Harvesting," in Magnetics, IEEE Transactions on, vol. 47, no. 10, pp. 2624-2627, Oct. 2011 doi:10.1109/TMAG.2011.2146233.

[5] Toba, A. and Lipo, T.A., "Generic torque-maximizing design methodology of surface permanent-magnet Vernier machine," IEEE Transactions on Industry Applications, vol. 36, no. 6, pp. 1539-1546, Nov/Dec 2000.

[6] K.-T. Chau, W. Li, and C. H. T. Lee, "Challenges and opportunities of electric machines for renewable energy," Progress in Electromagnetics Research B, Vol. 42, 45-74, 2012.

[7] Iwabuchi, N.; Kawahara, A.; Kume, T.; Kabashima, T.; Nagasaka, N., "A novel high-torque reluctance motor with rare-earth magnet," in Industry Applications, IEEE Transactions on, vol. 30, no. 3, pp. 609614, May/Jun 1994 doi:10.1109/28.293708

[8] M. A. Mueller and N. J. Baker, "Modelling the performance of the Vernier hybrid machine," in Proc. IEE - Electr. Power Appl., vol. 150, no. 6, pp. 647654, Nov. 2003.

[9] Yi Du; Chau, K. T.; Ming Cheng; Ying Fan; Yubin Wang; Wei Hua; Zheng Wang, "Design and Analysis of Linear Stator Permanent Magnet Vernier Machines," in Magnetics, IEEE Transactions on, vol.47, no.10, pp.4219-4222, Oct. 2011 doi:10.1109/TMAG.2011. 2156392

[10] Feng Xiao, Yi Du, Yubin Wang, Mu Chen, Ching, T. W. and Xianxing Liu, "Modeling and Analysis of a Linear Stator Permanent-Magnet Vernier HTS Machine," IEEE Transactions on Applied Superconductivity, vol. 25, no. 3, June 2015.

[11] Harris, M.R.; Pajooman, G.H.; Abu Sharkh, S.M., "The problem of power factor in VRPM (transverseflux) machines," in Electrical Machines and Drives, 1997 Eighth International Conference on (Conf. Publ. No. 444), vol., no., pp. 386-390, 1-3 Sep 1997 doi:10.1049/cp:19971104

[12] The Induction Machine Handbook: Ion Boldea and Syed A. Nasar CRC Press 2001

[13] Fei Zhao, Lipo, T. A. and Byung-il Kwon, "A Novel Dual-Stator Axial-Flux Spoke-Type Permanent Magnet Vernier Machine for Direct-Drive Applications," IEEE Transactions on Magnetics, vol. 50, no. 11, Nov. 2014

[14] Yi Du, Ming Cheng, K. T. Chau, Xianxing Liu, Feng Xiao, Wenxiang Zhao, Kai Shi and Lihong Mo, "Comparison of Linear Primary Permanent Magnet Vernier Machine and Linear Vernier Hybrid Machine," IEEE Transactions on Magnetics, vol. 50, no. 11, Nov. 2014.

[15] A. Toba and T. A. Lipo, "Novel dual-excitation permanent magnet vernier machine," in Proc. Conf. Rec. IEEE 34th IAS Annual Meeting, pp. 2539-2544, 1999.

[16] Yi Du, Chau, K.T., Ming Cheng, Ying Fan, Yubin 
Wang, Wei Hua and Zheng Wang, "Design and Analysis of Linear Stator Permanent Magnet Vernier Machines," IEEE Transactions on Magnetics, vol. 47, no. 10, pp. 4219-4222, Oct. 2011.

[17] A. Toba and T. A. Lipo, "Generic torque-maximizing design methodology of surface permanent-magnet vernier machine," in IEEE Transactions on Industry Applications, vol. 36, no. 6, pp. 1539-1546, Nov/Dec 2000. doi: $10.1109 / 28.887204$

[18] Z. Q. Zhu, D. Howe, E. Bolte and B. Ackermann, "Instantaneous magnetic field distribution in brushless permanent magnet DC motors. I. Open-circuit field," in IEEE Transactions on Magnetics, vol. 29, no. 1, pp. 124-135, Jan 1993. doi: 10.1109/20.195557

[19] T. A. Lipo, Introduction to AC Machine Design, 3rd ed. Madison, WI, USA: Univ. Wisconsin-Madison, 2007, pp. 95-101

[20] Boldea, I.; Nasar, S.A., "Linear electric actuators and generators," in Electric Machines and Drives Conference Record, 1997. IEEE International, vol., no., pp.MA1/1.1-MA1/1.5, 18-21 May 1997 doi: 10.1109/IEMDC.1997.604058

[21] Eastham, J.F., "Novel synchronous machines: linear and disc," in Electric Power Applications, IEE Proceedings B, vol. 137, no. 1, pp. 49-58, Jan 1990

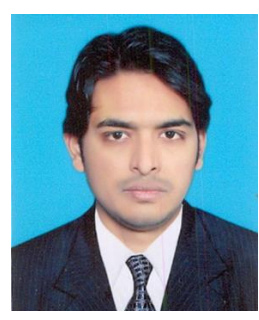

Salman Khaliq He was born in Punjab, Pakistan in 1986. He received his B.E and M.S in 2009 and 2012 from University of Engineering and Technology, Lahore and University of Management and Technology, Lahore, Pakistan, respectively. He worked in the capacity of Lab engineer as well as Lecturer in Electrical Engineering department for four years at University of Management and Technology, Lahore, Pakistan. Currently, he is working towards his Ph.D. degree in the Department of Electronic Systems Engineering at Hanyang University, Ansan, South Korea. His research interests include electromagnetic design of Electrical Machines.

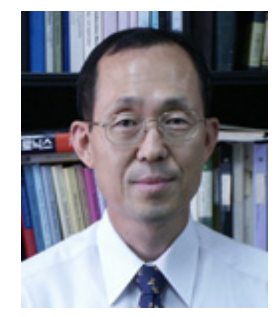

Byung-il Kwon He was born in 1956. $\mathrm{He}$ received his B.S. and M.S. in electrical engineering from Hanyang University, Ansan, Korea, and his Ph.D. in electrical engineering from the University of Tokyo, Tokyo, Japan, in 1989. He was a visiting researcher with the Faculty of Science and Engineering Laboratory, University of Waseda, Tokyo, from 1989 to 2000; a researcher with the Toshiba System Laboratory in 1990; a senior researcher with the Institute of Machinery and Materials Magnetic Train Business in 1991; and a visiting professor with the University of Wisconsin Madison, from 2001 to 2002 . He is currently a professor at Hanyang University. His research interests are design and control of electric machines. 